\title{
The Personal Knowledge Workbench of the NEPOMUK Semantic Desktop
}

\author{
Gunnar Aastrand Grimnes, Leo Sauermann, and Ansgar Bernardi \\ DFKI GmbH, Kaiserslautern, Germany \\ gunnar.grimnes@dfki.de, leo.sauermann@dfki.de, ansgar.bernardi@dfki.de
}

\begin{abstract}
In this paper we describe some of the personal information management features found in the PSEW prototype of the NEPOMUK Semantic Desktop project. Focussing on importing and adding new knowledge to the system as well as navigating, browsing, and searching the existing knowledge we show how a user can use NEPOMUK to integrate her information across application boundaries and create and discover new structures in her information space.
\end{abstract}

\section{The NEPOMUK Semantic Desktop}

The paper presents parts of the outcome of the now completed NEPOMUK project 1 . NEPOMUK aimed to create a specification for a Social Semantic Desktop and a prototype implementation of this specification. This paper will only describe the details around the prototype implementation, for information on the Semantic Desktop specifications such as the ontologies, the architecture blueprint and service descriptions we refer the reader to the project deliverables available from the project website as well as [12].

The NEPOMUK project resulted in two parallele implementations of the Semantic Desktop, one written in $\mathrm{C}++$ which is tightly integrated with the KDE4 desktop environment, and a cross platform version written in Java. This paper deals only with the Java version. Furthermore, we focus only the Personal Information Management (PIM) components of the NEPOMUK Semantic Desktop, that is, only the components used on a single desktop machine. NEPOMUK also includes social features allowing various kinds of distributed search and information sharing, but they will not be covered here.

The NEPOMUK approach to personal information management aims to overcome the limitation of having a separate applications for each type of data, such as email, photo-manager, publication-managers, etc. Instead all the information is integrated into one information-space and the user is free to relate and annotate information elements across application boundaries. The central hub for working with this information-space is called the NEPOMUK Personal Semantic Workbench (PSEW) and a typical view is show in Figure 1. Here we see a range of different views on the user's data, and a person concept, "Claudia",

${ }^{1}$ http://nepomuk.semanticdesktop.org

L. Aroyo et al. (Eds.): ESWC 2009, LNCS 5554, pp. 836 8402009.

(C) Springer-Verlag Berlin Heidelberg 2009 
being editted. The central idea for personal information management in NEPOMUK is semantic annotation, ranging from simple tagging to more sophisticated ontology based use of typed relations, class hierarchies, etc. Although semantically rich relations are possible in the framework, we have found the general hasTag relation to be at a level of complexity the users can easily understand and work with, and is also enough for most applications [3. In NEPOMUK we go beyond the simple text tags used by web2.0 sites such as del.icio.us or Flickr, and each tag is a PIMO concept; that is, tags belong to one of the classes in the Personal Information Modelling Ontology (PIMO). PIMO is the NEPOMUK way of letting the user express her world-model on the Semantic Desktop by extending and sub-classing the starting PIMO which provides general classes such as Document, Person, Company, etc. Users are free to get as involved as they wish, if they are happy with a flat list of tags, all tags can be left as the default pimo:Topic, or they can create a more elaborate structuring scheme. Automated services that will automatically assign the correct type to known cities, people and countries are also available.

From a technical point of view NEPOMUK works by adding all desktop knowledge into a central RDF store, paired with a Lucene index for full-text queries of the content of RDF literals 4 . We have defined a set of ontologies for representing desktop items, known collectively as NIE (Nepomuk Information Elements)2; NIE includes ontologies for contacts (Nepomuk Contact Ontology - NCO), messages (Messaging Ontology - NMO). The PIMO ontology is used to organise and interrelate this knowledge. A set of core information handling services, such as PIMO manipulation, data-crawling, etc., are available through HTTP based interfaces and a Eclipse RCP GUI is built in top of these. Additional plugins for existing applications are also built using the HTTP interfaces.

\section{Adding Knowledge}

When starting the use a new knowledge management tool, bootstrapping is an important problem. To the end-user, the value of the Semantic Desktop increases as more data is added, allowing richer interrelations and increased chances of finding interesting patterns.

In NEPOMUK the Aperture framework 3 is used for importing existing data into the Semantic Desktop. Aperture can crawl information from a wide range of sources, such as file systems, email servers, web2.0 web-sites and existing desktop applications such as MS Outlook or Mozilla Thunderbird. From the crawled information sources Aperture can extract meta-data as well as the textual contents of documents; most common document formats are supported, such MS Office documents, PDFs, HTML, JPEGs, PNGs, MP3s, other multi-media files, and many more.

When NEPOMUK is first installed the user has a choice of what data-sources to setup and crawl, common data-sources such as files and email are automati-

${ }^{2}$ http://www.semanticdesktop.org/ontologies/

${ }^{3}$ http://aperture.sourceforge.net 


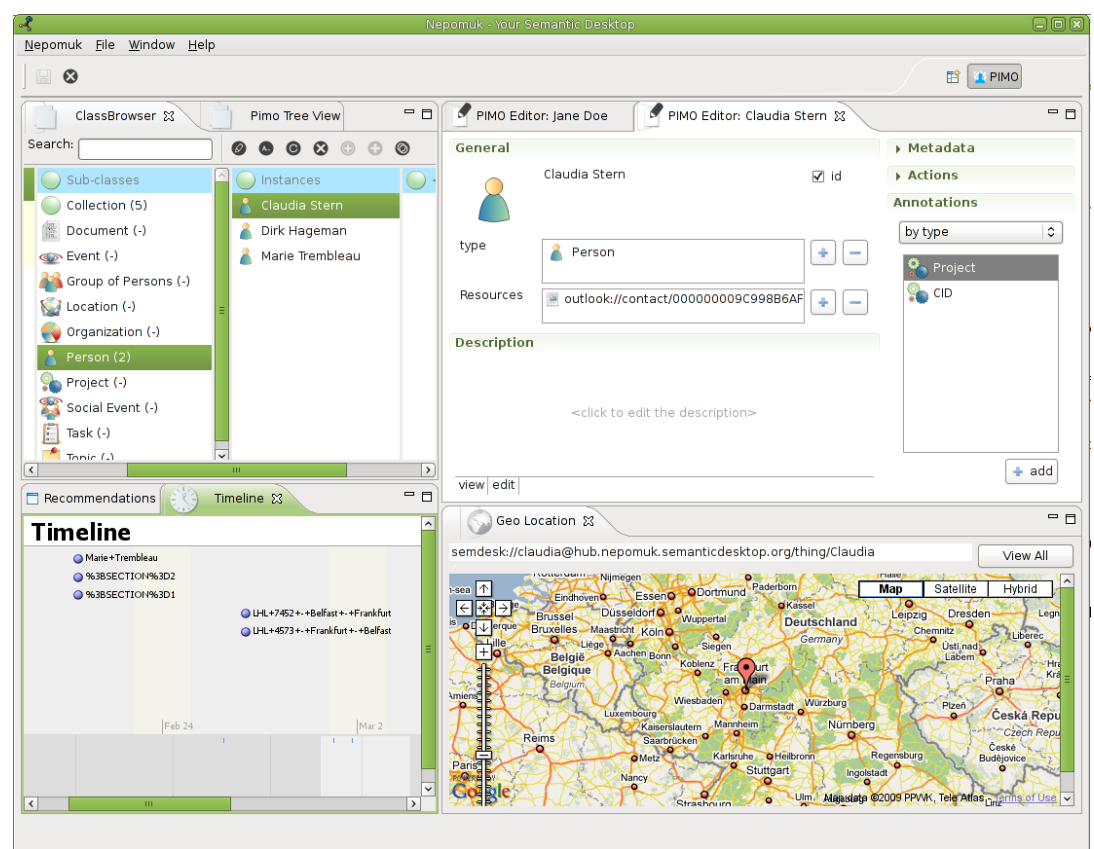

Fig. 1. The NEPOMUK Personal Semantic Workbench

cally detected by the system. Crawling is done regularly and iteratively, meaning changes to the original data will soon be picked up by NEPOMUK.

Once NEPOMUK has been setup and existing information has been crawled, NEPOMUK offers several ways to add new information directly into the Semantic Desktop data-cloud. Firstly, NEPOMUK includes a DropBox, a special folder that is monitored for changes, when a new file is downloaded from the web it can be saved in this folder. NEPOMUK will detect the file and analyse the content a pop-up a dialog with suggested tags, as shown in Figure 2. Here the file prototypearchitecture.pdf has been dropped, and the tags Marco Andriotti and Technical Workpackage Meeting has been proposed, the user himself has added a new tag: cidprototype. Each tag in NEPOMUK can be associated with a folder on disk, when a tag with an associated folder is assigned in the DropBox, the file is automatically moved to the appropriate location as well as tagged with the correct concept in one quick operation.

For content that is not downloaded into the DropBox NEPOMUK offers a bookmarklet for easy tagging of web-pages, as well as plugins for Mozilla Thunderbird and MS Outlook for tagging of emails.

\section{Information Enrichment and Annotation}

Importing the knowledge into a common RDF store only solves part of the information integration problem, as the data still forms separate islands for each 


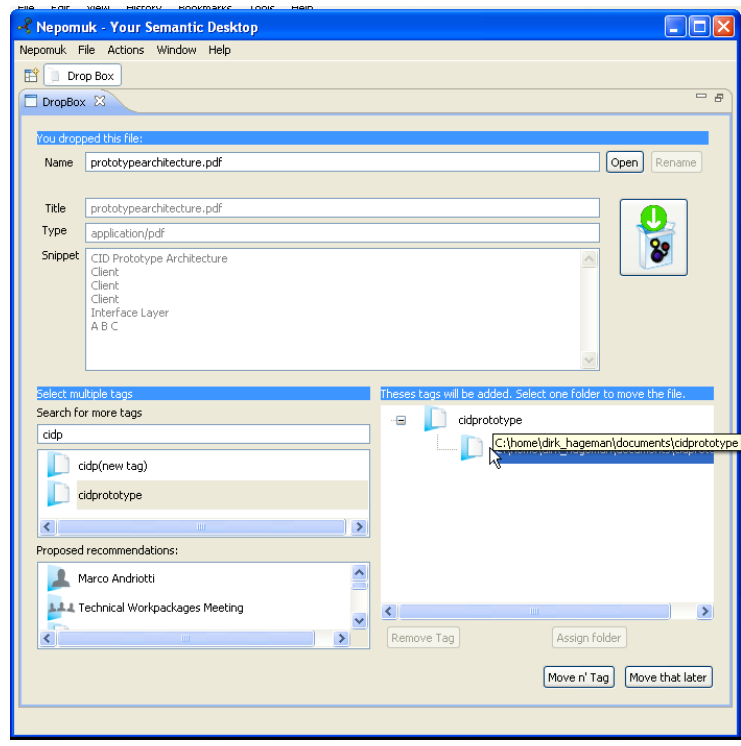

Fig. 2. The NEPOMUK DropBox

data-source. Creating the relations that for instance links the author of a PDF on disk to the sender of an email from Thunderbird is an important part of NEPOMUK and we call the process Local Data Alignment (LDA). Space does not allow us to go into details here, but NEPOMUK uses several heuristics for doing named entity merging; assigning the right class to known cities and countries; using the semantically enabled encyclopedia DBPedia 4 for finding additional information about concepts; and graph mining approaches for finding related resources. For crawled data, the data-alignment happens automatically. For manually created concepts the relations proposed by LDA are shows as recommendations in the user-interface.

\section{Browsing and Searching}

One of the key benefits of using Semantic Web technology to structure desktop information is that one is no longer tied to a single scheme of categorisation, i.e. in a traditional file-system a file resides in one folder, and one folder only. If one has chosen to arrange ones files by project, there is no way to later go back and view them by year. On the Semantic Desktop we can offer a wide range of views on the same data, for example, a timeline view that shows what concepts have been active in a certain period; a map view that shows the geographical location of concepts; a facetted browser; a tree-based view of the personal ontology, etc. For example, Figure 1 1shows the hierarchical class-browser for browsing concepts based on their class, a timeline view, a map view, as well as a concept being editted.

\footnotetext{
${ }^{4}$ http://dbpedia.org
} 
For the cases when browsing is not sufficient, NEPOMUK also offers a powerful search perspective, allowing the user to search her entire information space based either simple keyword queries as well as a more powerful structured query builder [5]. The structured query builder provides a visual interface for creating SPARQL queries by drag-dropping, and also offers heuristics for ontology relaxation when the exact ontology of the data queried is not known.

\section{Summary}

We have briefly outlined the features the NEPOMUK Semantic Desktop offers for personal information management, focussing on the process of adding information into the system and later browsing and re-finding this information. The NEPOMUK system we have discussed is open source, and the system, as well as the test-data and scripts that will be used for the demo, are all available from the project website.

\section{References}

1. Bernardi, A., Decker, S., van Elst, L., Grimnes, G., Groza, T., Handschuh, S., Jazayeri, M., Mesnage, C., Moeller, K., Reif, G., Sintek, M., Sauermann, L.: The Social Semantic Desktop: A New Paradigm Towards Deploying the Semantic Web on the Desktop. IGI Global (October 2008)

2. Groza, T., Handschuh, S., Moeller, K., Grimnes, G., Sauermann, L., Minack, E., Mesnage, C., Jazayeri, M., Reif, G., Gudjonsdottir, R.: The nepomuk project - on the way to the social semantic desktop. In: Proceedings of the Third International Conference on Semantic Technologies (I-SEMANTICS 2007), Graz, Austria (2007)

3. Sauermann, L., Heim, D.: Evaluating long-term use of the gnowsis semantic desktop for pim. In: Sheth, A., et al. (eds.) ISWC 2008. LNCS, vol. 5318, pp. 467-482. Springer, Heidelberg (2008)

4. Minack, E., Sauermann, L., Grimnes, G., Fluit, C., Broekstra, J.: The sesame lucene sail: Rdf queries with full-text search. Technical Report 2008-1, NEPOMUK Consortium (February 2008)

5. Kawase, R., Minack, E., Nejdl, W., Araäjo, S., Schwabe, D.: Incremental end-user query construction for the semantic desktop. In: International Conference on Web Information Systems and Technologies (WEBIST), Lisboa, Protugal (2009) 